\title{
Emergence and Persistence of Overweight and Obesity in 7- to 11-Year-Old Children
}

\author{
Naomi C. Bartle ad Claire Hill ${ }^{\text {b }}$ Laura Webber ${ }^{c}$ \\ Cornelia H.M. van Jaarsveld $^{d}$ Jane Wardle $^{d}$ \\ ${ }^{a}$ Coventry University, Priory Street, Coventry, ${ }^{b}$ Department of Psychology, Royal Holloway, \\ University of London, Surrey, ${ }^{c}$ National Heart Forum, ${ }^{d}$ Department of Epidemiology and \\ Public Health, University College London, London, UK
}

\section{Key Words}

Obesity · Adiposity · Growth · Child development · Longitudinal

\begin{abstract}
Aim: After dramatic rises in paediatric obesity, the critical period for obesity onset may now be pre-adolescence. Methods: We monitored adiposity over 4 years in 400 children aged 7-9 years recruited from schools in London. Weight, height, waist circumference (WC) and fat mass were measured annually. Weight status was defined using International Obesity Task Force (IOTF) criteria, and standardised scores and percentiles used British 1990 reference data. Results: BMI, WC and fat mass index all tracked strongly over time (average correlation for BMI $=0.95$ ). Emergence of obesity was relatively uncommon: only $2 \%$ of the total sample increased from overweight to obese over the 4-year period, and this was nearly matched by the $1.3 \%$ that reduced from obese to overweight. However, more children (6\%) moved from healthy weight to overweight than the reverse direction (2\%). There were greater absolute gains in adiposity in children with higher baseline weight status, but this was disguised in analyses using standardised scores. Obesity was not an emergent trait in middle childhood, but rates were already high and, in absolute terms, adiposity increased more in overweight and obese than healthy weight children. Conclusion: These results highlight the need for active management of obesity in middle childhood.
\end{abstract}

(c) 2013 S. Karger GmbH, Freiburg

\section{Introduction}

Adolescence has been long been identified as a 'critical period' for development of obesity [1-4], but more recent birth cohorts are showing a shift in the BMI growth curve, with children getting heavier at a younger age $[5,6]$. In a large adolescent sample followed from ages 11 to 
Bartle et al.: Emergence and Persistence of Overweight and Obesity in 7- to 11-Year-Old Children

16 years, there was little evidence for emergence of obesity, but rates were already high at age 11 years [7]. A 12-year follow-up of children born in the 1980s found the steepest increases in BMI were before and during puberty [8], and in a recent cohort, new incidence of obesity was highest between 7 and 11 years, with a strong tendency for it to persist into adolescence [9-11].

Given the evidence that pre-adolescence may be a high-risk time for emergence of obesity, it is important to understand growth patterns during this period. Few recent studies have tracked adiposity development closely across the pre-pubertal years, and those that have tend to report measurement intervals that span several years [e.g., 3, 8] except for the ALSPAC cohort, which is a very large sample of children born in 1991-1992 who are followed up annually. Furthermore, relatively few studies have indexed adiposity change in any way other than BMI; so there is less evidence about modern growth patterns for waist circumference (WC) or fat mass, although in ALSPAC all indices of adiposity indicated increasing rates of overweight and obesity from ages 7-11 years $[9,10]$.

As well as examining the emergence of obesity across childhood and adolescence, there is interest in whether obese children gain relatively more body fat than healthy-weight children. In the ALSPAC cohort, change in adiposity was indexed with standardised scores and indicated that obese children were more likely than healthy-weight children to lose BMI z-scores from ages 7-11 years, although they gained more body fat z-scores [9]. In a recent analysis of a US cohort, change in percentile scores for both BMI and body fat were lower in obese children, suggesting that obese children tended to get thinner, while normal-weight children got fatter [12]. However, because the absolute difference between adjacent standardised scores such as percentiles or z-scores is higher at the upper than the lower end of the weight distribution, use of standardised scores reduces the observed magnitude of adiposity change in heavier children. For this reason, raw BMI and WC scores are recommended to index change rather than standardised scores [13].

The present study is a secondary analysis of data collected for the Physical Exercise and Appetite in Children Study (PEACHES) that assessed the 'tracking' of BMI, WC and fat mass index (FMI) over 4 years in a cohort of children aged 7-9 years in 2006 (baseline) and examined change in adiposity over time by baseline weight status using both raw and standardised scores.

\section{Participants and Methods}

\section{Participants}

Parents of children in Years 3 and 4 (age 7-9 years) in five London schools $(\mathrm{N}=531$ ) were invited to allow their child to participate in a study of 'diet, activity and growth'. The majority $(\mathrm{N}=405,76 \%)$ gave consent for anthropometric measurements, which were obtained for 400 children at baseline, and for 333 children (82\%) on at least two further occasions. The sample size had been calculated in order to test associations between eating and activity phenotypes and future weight gain. Ethical approval was granted by the University College London Research Ethics Committee.

\section{Measures}

Date of birth, sex, ethnicity and postcode were obtained from school records. Each child's residential postcode was linked with an area-based index of socio-economic deprivation, the Townsend Index, which is derived from census data on car ownership, unemployment and overcrowded living conditions. Positive scores on this index represent greater deprivation while negative scores represent relative affluence [14]. For 46 pupils (11.5\%) the school had no record of postcode, and so the mean Townsend score for that child's school was used. Ethnicity was coded into four categories (White, Black, Asian, other), or for some analyses clustered into White and non-White.

Anthropometric measurements were collected by trained researchers using standard protocols. Height was measured to the nearest millimetre using a Leicester height measure (Seca, Birmingham, UK). The Tanita 
Bartle et al.: Emergence and Persistence of Overweight and Obesity in 7- to 11-Year-Old Children

TBF-300MA Body Composition Analyser (Tanita Corporation, Tokyo, Japan) was used to measure weight and fat mass to the nearest tenth of a kilogram. WC was measured approximately $4 \mathrm{~cm}$ above the umbilicus, to the nearest millimetre with a Seca 200 circumference measuring tape in accordance with recommendations [15]. Measurements were taken at baseline and then annually for 3 years (FU1, FU2, FU3), except that for approximately half the children $(n=163$ ) FU3 was brought forward by 3 or 6 months because of their imminent move to another school which would have resulted in loss to follow-up. Age is adjusted for in the analyses.

Data Treatment and Analyses

$\mathrm{BMI}$ and FMI were calculated as weight/height ${ }^{2}$ and fat mass $/$ height $^{2}$. Height, weight, BMI and WC were transformed into age- and sex-standardised scores (SDS) and percentiles based on British 1990 reference data using the Imsgrowth macro (http://homepage.mac.com/tjcole/ FileSharing1.html). The same software was used to categorise BMI into the International Obesity Task Force (IOTF) weight status categories: underweight, healthy weight, overweight and obese $[16,17]$. Intra-class correlation coefficients in a sub-sample of children assessed by two researchers $(n=30)$ showed high inter-rater reliability at all measurement occasions and for all adiposity indicators; at baseline these values were weight $(r=1.0)$, height $(r=0.99)$, BMI $(r=0.99)$, fat mass $(r=0.99)$ and WC $(r=0.88)$.

Associations between socio-demographic variables and baseline adiposity (BMI, FMI, WC) were examined using univariate ANOVA for categorical variables (ethnicity and sex) or Pearson correlation for continuous variables (deprivation). Changes in adiposity measures and weight status between baseline and final follow-up were examined with t-tests and chi-square analyses, respectively. Pearson correlations were calculated between baseline measurements and each follow-up (FU1, FU2, FU3). Chi-square analyses were used to examine the stability of weight status between baseline and FU3. In order to examine change in adiposity relative to baseline IOTF status, raw BMI, WC and FMI scores were adjusted by subtracting the median change for each child's age and sex group from their own change score, as recommended [13]. Univariate ANOVA with repeated contrasts was used to compare change in adiposity across weight status groups for these adjusted scores, SD scores and percentile scores.

Multilevel, linear, mixed models with measurement occasion as a level 1 factor and child as a level 2 factor were used for all adiposity measures. There was no evidence of clustering within schools, so school was not defined as a further level. MLwiN software does not require data from every child at every visit, and therefore maximised the available data [18]. Because the four measurement occasions were not equidistant for each child (some were measured early at the final follow-up), age at measurement was included in the models. Sex, ethnicity and deprivation were included as unique predictor variables as well as testing each two-way interaction with age, and the three-way interactions with age. We used 'raw' adiposity measures (BMI, WC and FMI) as well as standardised scores (BMI SDS, WC SDS) in these analyses for comparison.

\section{Results}

Of the 400 children measured at baseline, data were collected on 347 at FU1 (87\%), 328 at FU2 (82\%), and 302 at FU3 (76\%). Missing data were due to children leaving the school $(n=77)$ or being absent on the days of testing $(n=21)$. The mean Townsend Index was high, indicating the sample was relatively deprived. Other sample characteristics at baseline and follow-up are shown in table 1. Age, area-level deprivation, BMI, WC, fat mass and FMI did not differ between completers and non-completers. There was also no association between completer status and sex, ethnicity or baseline weight status (data not shown).

At baseline, boys and girls did not differ significantly in BMI or WC, but girls' FMI (3.40 $\mathrm{kg} / \mathrm{m}^{2}, \mathrm{SD}=2.08$ ) was higher than boys' $\left(3.02 \mathrm{~kg} / \mathrm{m}^{2}, \mathrm{SD}=1.68 ; \mathrm{F}(1,395)=3.98, \mathrm{p}=0.047\right)$. Deprivation was not significantly correlated with any of the adiposity measures (all $r<0.10$, all $\mathrm{p}>0.10)$. Black children had the highest mean FMI ( $\left.3.86 \mathrm{~kg} / \mathrm{m}^{2}, \mathrm{SD}=2.30\right)$, Asian children's FMI was $3.32 \mathrm{~kg} / \mathrm{m}^{2}(\mathrm{SD}=1.74)$, and White children had the lowest FMI $\left(2.87 \mathrm{~kg} / \mathrm{m}^{2}\right.$, $\mathrm{SD}=1.60, \mathrm{~F}(3,375)=6.37, \mathrm{p}<0.01)$. Ethnic differences for WC showed a similar pattern $(F(3,378)=3.36, p=0.02)$, as did BMI although the difference was not significant in this case $(\mathrm{F}(3,378)=2.33, \mathrm{p}=0.07)$. 
Bartle et al.: Emergence and Persistence of Overweight and Obesity in 7- to 11-Year-Old Children

Table 1. Sample characteristics at baseline and each follow-up

\begin{tabular}{|c|c|c|c|c|c|}
\hline & $\begin{array}{l}\text { Baseline } \\
(n=400)\end{array}$ & $\begin{array}{l}\text { FU1 } \\
(n=347)\end{array}$ & $\begin{array}{l}\text { FU2 } \\
(n=328)\end{array}$ & $\begin{array}{l}\text { FU3 }^{a} \\
(n=302)\end{array}$ & $\begin{array}{l}\text { Baseline to FU3 } \\
\text { change }\end{array}$ \\
\hline \multicolumn{6}{|l|}{ Sex, \% (n) } \\
\hline Boys & $51.5(206)$ & $51.0(177)$ & $51.2(168)$ & $50.7(153)$ & - \\
\hline Girls & $48.5(194)$ & $49.0(170)$ & $48.8(160)$ & $49.3(149)$ & - \\
\hline \multicolumn{6}{|l|}{ Ethnicity, \% (n) } \\
\hline White & $43.4(174)$ & $45.2(157)$ & $45.4(149)$ & $47.4(143)$ & - \\
\hline Black & $26.2(105)$ & $28.2(98)$ & $28.7(94)$ & $27.5(83)$ & - \\
\hline Asian & $14.2(57)$ & $14.4(50)$ & $13.7(45)$ & $13.6(41)$ & - \\
\hline Other & $11.7(47)$ & $12.1(42)$ & $12.2(40)$ & $11.6(35)$ & - \\
\hline Missing & $4.5(17)$ & 0 & 0 & 0 & - \\
\hline Deprivation (mean \pm SD) & $4.31 \pm 3.50$ & $4.31 \pm 3.61$ & $4.30 \pm 3.62$ & $4.38 \pm 3.57$ & - \\
\hline Age, years (mean $\pm S D)$ & $8.28 \pm 0.64$ & $9.25 \pm 0.62$ & $10.25 \pm 0.62$ & $11.05 \pm 0.46$ & $\mathrm{t}(301)=194.9^{*}$ \\
\hline \multicolumn{5}{|c|}{ IOTF Weight Category, \% (n) } & $\chi^{2}(9)=358.3^{*}$ \\
\hline Underweight & $16.0(64)$ & $12.4(43)$ & $14.7(48)$ & $13.2(40)$ & \\
\hline Healthy weight & $65.3(261)$ & $64.7(224)$ & $61.5(201)$ & $64.9(196)$ & \\
\hline Overweight & $13.5(54)$ & $17.3(60)$ & $17.4(57)$ & $16.2(49)$ & \\
\hline Obese & $5.3(21)$ & $5.2(18)$ & $6.4(21)$ & $5.6(17)$ & \\
\hline \multicolumn{6}{|l|}{ Adiposity, mean \pm SD } \\
\hline BMI, $\mathrm{kg} / \mathrm{m}^{2}$ & $16.53 \pm 2.64$ & $17.32 \pm 3.01$ & $17.98 \pm 3.33$ & $18.44 \pm 3.54$ & $\mathrm{t}(301)=21.4^{*}$ \\
\hline BMI SDS & $0.13 \pm 1.35$ & $0.22 \pm 1.28$ & $0.25 \pm 1.33$ & $0.23 \pm 1.36$ & $\mathrm{t}(301)=3.5^{*}$ \\
\hline $\mathrm{WC}, \mathrm{cm}$ & $60.05 \pm 6.33$ & $62.35 \pm 7.23$ & $64.76 \pm 8.17$ & $66.22 \pm 8.66$ & $t(299)=23.1^{*}$ \\
\hline WC SDS & $0.94 \pm 1.04$ & $1.00 \pm 1.04$ & $1.04 \pm 1.07$ & $0.98 \pm 1.07$ & $\mathrm{t}(299)=1.5 \mathrm{~ns}$ \\
\hline Fat mass, kg & $5.63 \pm 3.59$ & $7.01 \pm 4.61$ & $8.04 \pm 5.42$ & $8.82 \pm 6.03$ & $\mathrm{t}(297)=12.7^{*}$ \\
\hline FMI, $\mathrm{kg} / \mathrm{m}^{2}$ & $3.20 \pm 1.89$ & $3.64 \pm 2.21$ & $3.84 \pm 2.43$ & $3.96 \pm 2.57$ & $\mathrm{t}(297)=10.4^{*}$ \\
\hline
\end{tabular}

\section{Change in Adiposity}

As expected, between baseline and FU3, all three measures of adiposity increased significantly (table 1). BMI SDS also increased, indicating that children grew faster on average than the 1990 reference population, although WC SDS did not change significantly from baseline. Means from children who had data at all four measurement times showed similar increases to those who had some missing data (data not shown).

All adiposity measures showed strong tracking over time. BMI and FMI scores were correlated at or above $r=0.89$ between all occasions (all $p<0.001$ ) and correlations for WC were marginally lower $(0.81-0.90$, all $\mathrm{p}<0.001)$.

\section{Emergence and Remission of Overweight/Obesity}

At baseline, $13.5 \%$ of the children were overweight and a further $5.3 \%$ were obese (total overweight and obese $=18.8 \%$ ). Over follow-up there was a small increase in prevalence of overweight and obesity combined, reaching $21.8 \%$ at FU3, with a similar decrease in prevalence of underweight (down from $16.0 \%$ to $13.2 \%$; see table 1 ).

The majority of children (80\%) remained in the same IOTF category throughout the study, and weight status at baseline was strongly associated with weight status at FU3 ( $\chi^{2}(9)$ $=356.19, \mathrm{p}<0.001$ ) (tables 1, 2). Across the whole sample, 6.0\% moved up from healthy weight to overweight (none from healthy weight to obese), and $2.3 \%$ moved down from overweight to healthy weight (none from overweight to underweight). 
Bartle et al.: Emergence and Persistence of Overweight and Obesity in 7- to 11-Year-Old Children

Table 2. Weight status at baseline and follow-up 3 for completers ${ }^{\mathrm{a}}$

\begin{tabular}{|c|c|c|c|c|c|c|c|c|}
\hline \multirow[t]{3}{*}{ Baseline IOTF category } & \multicolumn{8}{|c|}{ FU3 IOTF category } \\
\hline & \multicolumn{2}{|c|}{ underweight } & \multicolumn{2}{|c|}{ healthy weight } & \multicolumn{2}{|c|}{ overweight } & \multicolumn{2}{|c|}{ obese } \\
\hline & $\mathrm{n}$ & $\%$ & $\mathrm{n}$ & $\%$ & $\mathrm{n}$ & $\%$ & $\mathrm{n}$ & $\%$ \\
\hline Underweight, $\mathrm{n}=49$ & 30 & 61.2 & 19 & 38.8 & 0 & 0 & 0 & 0 \\
\hline Healthy weight, $n=197$ & 10 & 5.1 & 169 & 85.8 & 18 & 9.1 & 0 & 0 \\
\hline Overweight, $n=40$ & 0 & 0 & 7 & 17.5 & 27 & 67.5 & 6 & 15.0 \\
\hline Obese, $n=16$ & 0 & 0 & 1 & 6.3 & 4 & 25.0 & 11 & 68.8 \\
\hline
\end{tabular}

IOTF = International Obesity Task Force.

${ }^{\mathrm{a}} \mathrm{N}=302$, all those with data at baseline and FU3.

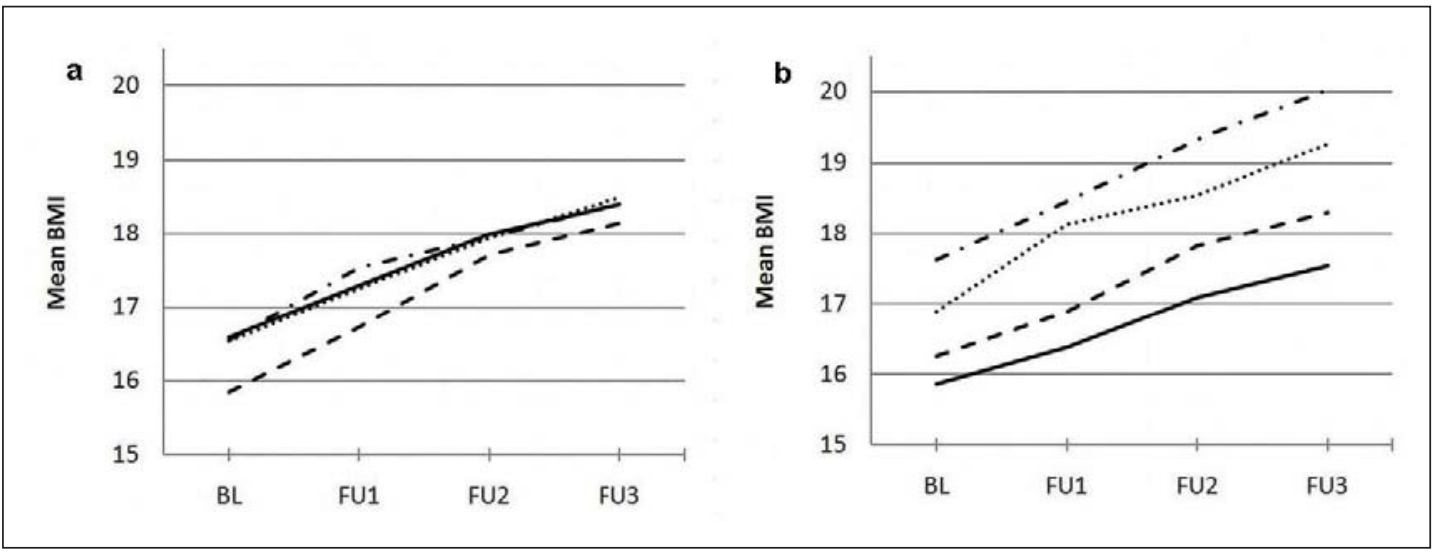

Fig. 1. Change in BMI by ethnicity and sex. a Males. b Females. Presenting observed, unadjusted means for children with complete data (males $n=146$, females $n=144$ ).

Of those who were overweight at baseline, $68 \%$ remained overweight, $18 \%$ were healthy weight and 15\% were obese at FU3 (table 2). Of those who were obese at baseline, $69 \%$ were still obese, $25 \%$ had become overweight, and $6 \%(\mathrm{n}=1)$ became healthy weight at FU3. Of those in the healthy-weight category at baseline, $86 \%$ were still healthy weight with $9 \%$ becoming overweight and $5 \%$ becoming underweight at FU3.

\section{Influence of Socio-Demographic Variables on Longitudinal Trends in Adiposity}

We used mixed models to test the independent effects of sex, ethnicity and deprivation on growth trajectories. BMI increased on average by $0.69 \mathrm{~kg} / \mathrm{m}^{2}(95 \%$ CI beta $0.62-0.77$ $\mathrm{kg} / \mathrm{m}^{2}$ ) a year, with no main effect of sex. There was a significant three-way interaction between sex, ethnicity and age, with Black girls increasing their BMI by $0.21 \mathrm{~kg} / \mathrm{m}^{2}(0.09-$ $0.33 \mathrm{~kg} / \mathrm{m}^{2}$ ) more a year than White girls (illustrated in fig. 1B). A similar pattern emerged for WC: on average, it increased by $2.26 \mathrm{~cm}(1.99-2.53 \mathrm{~cm})$ a year, but Black girls' WC increased by $0.51 \mathrm{~cm}(0.29-0.73 \mathrm{~cm})$ more a year than White girls' WC. FMI increased by $0.12 \mathrm{~kg} / \mathrm{m}^{2}$ $\left(0.04-0.20 \mathrm{~kg} / \mathrm{m}^{2}\right)$ a year. Girls increased in their FMI by $0.16 \mathrm{~kg} / \mathrm{m}^{2}\left(0.08-0.24 \mathrm{~kg} / \mathrm{m}^{2}\right)$ more a year than boys, and black girls increased by $0.11 \mathrm{~kg} / \mathrm{m}^{2}\left(0.01-0.21 \mathrm{~kg} / \mathrm{m}^{2}\right)$ a year more than White girls. The models for WC SDS and BMI SDS showed similar trends, with black girls showing the greatest gains in adiposity. 
Bartle et al.: Emergence and Persistence of Overweight and Obesity in 7- to 11-Year-Old Children

Table 3. Changes in three different indices of adiposity, using raw and standardised scores between baseline and FU3, by baseline weight status

\begin{tabular}{llllll}
\hline \multirow{2}{*}{ Change in } & \multicolumn{2}{l}{ Baseline IOTF category, mean (SD) } & \multirow{2}{*}{ ANOVA $^{\mathrm{b}}$} \\
\cline { 2 - 5 } & underweight & healthy weight & overweight $^{\mathrm{a}}$ & obese & \\
\hline Adjusted BMI & $-0.52(0.95)$ & $0.08(1.29)$ & $1.37(2.21)^{*}$ & $1.51(2.36)$ & $\mathrm{F}(3,298)=17.00^{* * *}$ \\
Adjusted WC & $-0.28(0.58)$ & $0.06(1.03)$ & $0.97(1.79)^{*}$ & $1.37(1.71)$ & $\mathrm{F}(3,294)=14.95^{* * *}$ \\
Adjusted FMI & $-0.68(2.98)$ & $0.09(4.23)$ & $2.91(6.57)^{*}$ & $4.45(6.64)$ & $\mathrm{F}(3,296)=9.20^{* * *}$ \\
BMI SDS & $0.18(0.66)$ & $0.20(0.55)$ & $0.19(0.54)$ & $-0.09(0.40)$ & $\mathrm{F}(3,298)=1.28$ \\
WC SDS & $0.11(0.75)$ & $0.08(0.68)$ & $0.03(0.61)$ & $-0.14(0.55)$ & $\mathrm{F}(3,297)=0.61$ \\
BMI centile & $6.60(13.03)$ & $6.38(17.07)$ & $0.10(8.31)^{*}$ & $-0.52(1.68)$ & $\mathrm{F}(3,298)=2.83^{*}$ \\
WC centile & $2.91(21.57)$ & $0.53(18.94)$ & $-1.27(6.84)$ & $-0.26(0.62)$ & $\mathrm{F}(3,297)=0.43$ \\
\hline
\end{tabular}

${ }^{a}$ Significance refers to contrast between healthy weight and overweight category ${ }^{*} \mathrm{p}<0.05$.

${ }^{b}$ Significance refers to overall ANOVA ${ }^{*} \mathrm{p}<0.05,{ }^{* *} \mathrm{p}<0.01,{ }^{* * *} \mathrm{p}<0.001$.

Fig. 2. Adjusted change in BMI from baseline to follow-up 3, according to baseline IOTF weight category.

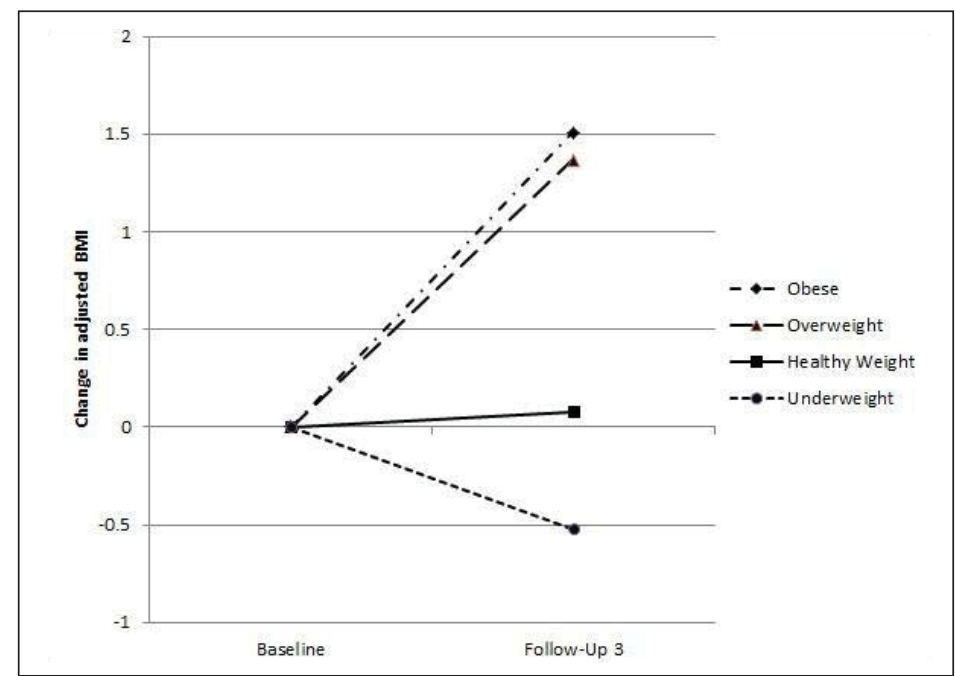

\section{Change in Adiposity by Baseline Weight Status}

Based on absolute values, children who were obese at baseline increased by $17.89 \mathrm{~kg}$ in total weight, $3.1 \mathrm{~kg} / \mathrm{m}^{2}$ in BMI, $1.8 \mathrm{~kg} / \mathrm{m}^{2}$ in FMI and $9.8 \mathrm{~cm}$ in WC. Overweight children had similar increases: $16.38 \mathrm{~kg}$ (total weight), $3.1 \mathrm{~kg} / \mathrm{m}^{2}$ (BMI), $1.5 \mathrm{~kg} / \mathrm{m}^{2}$ (FMI), and $8.6 \mathrm{~cm}$ (WC). In comparison, healthy-weight children had much smaller increases: $11.29 \mathrm{~kg}$ (total weight), $1.8 \mathrm{~kg} / \mathrm{m}^{2}$ (BMI), $0.6 \mathrm{~kg} / \mathrm{m}^{2}$ (FMI) and $5.8 \mathrm{~cm}$ (WC).

Three indices of change in adiposity were compared: change in adjusted raw scores, change in SDS as recommended [13], and change in percentiles (table 3). The adjusted raw scores indicate a clear trend for increasing gains in adiposity with increasing baseline IOTF status (fig. 2). Univariate ANOVAs and repeated contrasts showed a significant difference in growth rate between healthy-weight and overweight/obese groups for BMI, FMI and WC (table 3), with greater increases in adiposity in the overweight and obese, and also increasing variability especially for WC.

In contrast, using either SDS or percentiles for BMI and WC indicated that children in higher IOTF categories at baseline appeared to either increase less or actually decrease in 
Bartle et al.: Emergence and Persistence of Overweight and Obesity in 7- to 11-Year-Old Children

adiposity compared to children in the healthy-weight and underweight categories. Table 3 shows that the underweight, healthy-weight and overweight groups show similar levels of change in SDS while the obese group show a decrease in SDS between baseline and FU3 (although the ANOVA was not significant for either BMI or WC). A similar pattern occurred with percentiles, with overweight children showing significantly less increase in BMI between baseline and FU3 than healthy-weight children.

\section{Discussion}

We assessed adiposity annually over 4 years in a school-based sample of 400 children to assess whether the pre-pubertal period is a high-risk time for development of obesity. We found little evidence for a significant increase in the number of children meeting the IOTF criterion for obesity over the pre-pubertal years. There were 6 new cases of obesity, all of whom had been overweight at baseline, but this was matched by a similar number reducing from obese to overweight or healthy weight $(n=5)$. The incidence of new cases of overweight and obesity appeared lower than in a much larger study of 7- to 11-year-olds, in which $6.7 \%$ of children who were of healthy weight at baseline became obese and $11.8 \%$ became overweight (in comparison to $0 \%$ and $9 \%$ in our study), but this study used different cut-offs (85th and 95th percentiles) [10].

Although new cases of obesity were relatively infrequent, our results gave little reassurance that obese pre-adolescent children 'grow out of' excess adiposity. Almost all children who were overweight or obese when they were first measured at around age 7-8 years remained so 3 years later; consistent with data from a larger tracking study over a similar age period [9]. Furthermore, the prevalence of overweight increased due to more healthy-weight children moving up to overweight than overweight children moving down to healthy weight.

In contrast to some previous studies where higher socio-economic status was protective against development of overweight [19], we found no association with area-level deprivation. However, area-level markers of socio-economic status are likely to be less sensitive than markers that are closer to the individual environment such as parental education. There were ethnic differences in adiposity. Black children had the highest levels of adiposity and white children the lowest levels, with Asian children in between, and black girls had the steepest increases in adiposity. These findings confirm previous findings on ethnic differences $[20,12]$, extending them across three different indices of adiposity. The causes of ethnic differences are not well understood, but greater declines in physical activity have been described among black than white girls [20], and black adolescents in the UK have poorer dietary practices [21]. Future work should address the cultural determinants of eating and activity levels.

This study was novel in examining adiposity gains by baseline weight status and importantly by comparing raw and standardised scores as indices of change. On the basis of changes in standardised scores (SDS or percentiles), obese children showed lower increases in adiposity than other children. In contrast, using raw scores adjusted for age and sex indicated larger gains in adiposity for the overweight and obese children. The pattern observed for the adjusted raw scores is likely to reflect the increasing divergence of the weight distribution that can be observed on growth curves during the pre-pubertal period [22] whereas SDS and percentiles artificially attenuate the observed growth in larger children, as observed in another study of younger children [13]. This might explain the contrasting findings observed in the literature with regard to adiposity change in this age group [e.g., 8, 12]. Research into the best way to monitor change in adiposity in children is needed to avoid any further confusion in the literature. 
Weight status is known to track from childhood [23], and therefore many of the obese children in our sample are likely to become obese adults; given the early age of onset, the risks to health are substantial [24]. Weight management interventions should therefore be considered for obese children as young as 7 years in view of the increasing body of evidence that they are unlikely to return to a healthy weight spontaneously. Even overweight children could be an important target group for intervention because, with an average gain in adiposity between childhood and adulthood, they are at risk of obesity in the future. What these results also show is that obese children gain more in terms of weight, BMI, body fat and WC than healthy-weight children; so their health risk is progressively increasing.

One limitation of this study is that the observed rates of overweight and obesity $(22 \%$ at age 11) were somewhat lower than national figures that are closer to $27 \%$ [25]. We suspect that heavier children were less likely to enter the study; but once in the study, they were no more likely to be lost to follow-up, so the tracking results are likely to be valid. Otherwise, the sample is broadly representative of the population of London in terms of ethnic diversity and socio-economic status. A second limitation was the high ethnic diversity in a relatively small sample. This was designed to maximise generalizability, but it meant that we did not have a large enough samples for ethnic sub-group comparisons. Finally, in this age group, it would be interesting in future studies to account for pubertal level when interpreting patterns of growth.

\section{Conclusion}

We demonstrated transitions from healthy weight to overweight, but no rise in rates of obesity over the pre-adolescent years. Obese children gained more body fat than healthyweight children. The fact that obesity rarely resolved and the fat burden increased in obese children, emphasises the importance of early intervention. We also demonstrated that use of SDS or percentiles for indexing change in adiposity underestimated growth in larger children relative to use of raw scores, highlighting the need for consensus on the best way to monitor adiposity change in this age group.

\section{Acknowledgements}

We are grateful for the contribution of schools and children who participated in the study and the statistical advice from David Boniface. The PEACHES study is supported by Cancer Research UK (grant C1418/ A6142).

NB conducted the analysis and interpretation of the data and wrote the paper. CH and LW collected the data. CvJ advised on the analysis and interpretation. JW conceived of the study and advised on the analysis and interpretation. All authors have contributed to the writing of the manuscript and have critically reviewed the contents.

\section{Disclosure Statement}

The authors declare no conflict of interest. 
Bartle et al.: Emergence and Persistence of Overweight and Obesity in 7- to 11-Year-Old Children

\section{References}

$>1$

2

$>3$

\section{Nutr 1999;70:145s-148s.}

4 Guo SS, Huang C, Maynard LM, et al: Body mass index during childhood, adolescence and young adulthood in relation to adult overweight and adiposity: the FELS Longitudinal Study. Int J Obes Relat Metab Disord. 2000; 24:1628-1635.

-5 Lee JM, Pilli S, Gebremariam A, et al: Getting heavier, younger: trajectories of obesity over the life course. Int J Obes (Lond) 2010;34:614-623.

$\checkmark 6$ Johnson W, Soloway LE, Erickson D, et al: A changing pattern of childhood BMI growth during the 20th century: 70 y of data from the Fels Longitudinal Study. Am J Clin Nutr. 2012;95:1136-1143.

$>7$ Wardle J, Henning Brodersen N, Cole TJ, Jarvis MJ, Boniface DR: Development of adiposity in adolescence: five year longitudinal study of an ethnically and socioeconomically diverse sample of young people in Britain. BMJ 2006;332:1130-1135.

$>8$ Rzehak P, Heinrich J: Development of relative weight, overweight and obesity from childhood to young adulthood. A longitudinal analysis of individual change of height and weight. Eur J Epidemiol 2006;21:661672.

-9 Wright CM, Emmett PM, Ness AR, Reilly JJ, Sherriff A: Tracking of obesity and body fatness through midchildhood. Arch Dis Child 2010;95:612-617.

$>10$ Hughes AR, Sherriff A, Lawlor DA, Ness AR, Reilly JJ: Incidence of obesity during childhood and adolescence in a large contemporary cohort Prev Med 2011;52:300-304.

$>11$ Hughes AR, Sheriff A, Lawlor DA, Ness AR, Reilly JJ: Timing of excess weight gain in the Avon Longitudinal Study of Parents and Children (ALSPAC) Pediatrics 2011;127:e730-e736.

12 Williamson DA, Han H, Johnson WD, Stewart TM, Harsha DW: Longitudinal study of body weight changes in children: who is gaining and who is losing weight Obesity (Silver Spring) 2011;19:667-670.

13 Cole TJ, Faith MS, Pietrobelli A, Heo M: What is the best measure of adiposity change in growing children: BMI, BMI \%, BMI z-score or BMI centile? Eur J Clin Nutr 2005;59:419-425.

14 Townsend P, Phillimore P, Beattie A: Health and Deprivation: Inequality in the North. London, Croon Helm, 1988.

15 Rudolf M, Walker J, Cole TJ: What is the best way to measure waist circumference? Int J Pediatr 0bes 2007;2: 58-61.

16 Cole TJ, Bellizzi MC, Flegal KM, Dietz WH: Establishing a standard definition for child overweight and obesity worldwide: international survey. BMJ 2000;320:1240-1243.

$\checkmark 17$ Cole TJ, Flegal KM, Nicholls D, Jackson AA: Body mass index cut offs to define thinness in children and adolescents: international survey BMJ 2007;335:194-197.

18 Rasbash J, Charlton C, Browne WJ, Healy M, Cameron B: MLwiN Version 2.02. Centre for Multilevel Modelling, University of Bristol, 2005.

-19 Semmler C, Ashcroft J, van Jaarsveld CHM, Carnell S, Wardle J: Development of overweight in children in relation to parental weight and socioeconomic status. Obesity (Silver Spring) 2009;17:814-820.

-20 Freedman DS, Kettel Khan L, Serdula MK, Dietz WH, Srinivasan SR, Berenson GS: Racial differences in the tracking of childhood BMI to adulthood. Obes Res 2005;13:928-934.

-21 Harding S, Teyhan A, Maynard MJ, Cruickshank JK: Ethnic differences in overweight and obesity in early adolescence in the MRC DASH study: the role of adolescent and parental lifestyle. Int J Epidemiol 2008;37: 162-172.

-22 Cole TJ, Freeman JV, Preece MA: Body mass index reference curves for the UK, 1990 Arch Dis Child 1995;73: 25-29.

-23 Deshmukh-Taskar P, Nicklas TA, Morales M, Yang S-J, Zakeri I, Berenson GS: Tracking of overweight status from childhood to young adulthood: the Bogalusa Heart Study. Eur J Clin Nutr 2006;60:48-57.

24 Maffeis C, Tato L: Long-term effects of childhood obesity on morbidity and mortality. Horm Res 2001;55(suppl 1):42-45.

25 Stamatakis E, Zaninotto P, Falaschetti E, Mindell J, Head J: Time trends in childhood and adolescent obesity in England from 1995 to 2007 and projections of prevalence to 2015. J Epidemiol Community Health 2010;64: 167-174. 